\title{
Telemedicine in Cardiology Outpatient Clinic: First Experience from a Tertiary Medical Center During the COVID-19 Pandemic
}

\author{
๑ Elton Soydan, ๑ Meral Kayıkçığlu, ๑ Mehdi Zoghi
}

Ege University School of Medicine, Department of Cardiology, İzmir, Turkey

\begin{abstract}
Objectives: A pronounced spread of coronavirus disease-2019 (COVID-19) all over the world has led to unpredictable overfilling of medical facilities, thus opening the way for the implication of digital health. We aimed at assessing firsttime experience of telemedicine (TM) in the cardiology outpatient clinic of a tertiary medical center during the pandemic.

Materials and Methods: TM was used in the cardiology outpatient clinic from April $7^{\text {th }}$ to May $29^{\text {th }}, 2020$. All the patients that had applied to TM were included in the study. TM was performed by phone calls. The data written by the cardiologist telecommunicating with the patients were recorded by accessing each patient's electronic file. Assessment of TM in terms of efficacy and patient satisfaction was made in August 2020 by recalling all the patients by phone.

Results: A total of 140 patients had TM visit on the appointment day. The population was older $(69 \pm 13.75)$ with a male predominance $(53.5 \%)$. Main complaints were chest pain and high blood pressure. Twenty patients had their medical drugs rearranged. Forty-two patients were invited for face-to-face cardiac evaluation. One of them was hospitalized due to heart failure. Almost all (93.6\%) were very satisfied and preferred TM instead of face-to-face outpatient clinic service in terms of easy complaint expression and easy understanding of medication rearrangement (53.6\%). They stated that TM was efficient in the prevention of unnecessary hospital visits and it should continue after the pandemic as well.

Conclusion: TM is a potential alternative for continuing healthcare delivery to most of the cardiac patients during the COVID-19 pandemic. Future research comparing other digital health tools is needed to accurately assess its use in cardiological care.
\end{abstract}

Keywords: Telemedicine, COVID-19 pandemic, digital health, healthcare

Address for Correspondence: Elton Soydan, Ege University School of Medicine, Department of Cardiology, İzmir, Turkey e-mail: eltonsoydan@hotmail.com ORCID: orcid.org/0000-0002-9897-5484

Received: 30.01.2021 Accepted: 07.04.2021

Cite this article as: Soydan E, Kayıkçığlu M, Zoghi M. Telemedicine in Cardiology Outpatient Clinic: First Experience from a Tertiary Medical Center During the COVID-19 Pandemic. EJCM 2021;9(2):83-93.

DOI: 10.32596/ejcm.galenos.2021-01-08

${ }^{\circ}$ Copyright 2021 by Heart and Health Foundation of Turkey (TÜSAV) / E Journal of Cardiovascular Medicine published by Galenos Publishing House. 


\section{Introduction}

With abrupt onset of the pandemic, steeply increasing number of patients with coronavirus disease-2019 (COVID-19) caused a great pressure on hospitals and healthcare systems. In order to respond to the rapidly increasing number of COVID-19 patients, all elective medical procedures were cancelled in hospitals and outpatient clinics were suspended to reduce the risk of contraction of the virus ${ }^{(1,2)}$.

However, all cardiovascular patients pose high risk to COVID-19 infection and certainly its course could be more disastrous with higher mortality and morbidity risk ${ }^{(3)}$. Therefore, health care facilities adapted themselves by introducing digital health care in order to limit the unnecessary exposure of both the patients and health care providers to corona virus ${ }^{(4)}$. Digital health defined as "collection, sharing and manipulation of digital information to improve patient health and health care delivery" has been recently provided by telecommunication technology (telemedicine) in order to deliver health care to patients not able to be admitted to hospitals ${ }^{(5)}$. Telemedicine (TM) has proved efficient towards cardiovascular patient management with considerable good outcomes in terms of mortality and morbidity ${ }^{(6,7)}$. In the light of the aforementioned prevention modalities, TM was introduced into our cardiology outpatient clinic by the beginning of April 2020. TM, used for the first time in our cardiology department, brought up multiple questions in terms of applicability and efficiency towards patients' management of cardiovascular diseases. In addition, there is a blank in the evaluation of the new digital health modalities during the pandemic period. Therefore, we aimed to accurately assess the efficiency of TM communication technology and describe the patient complaints, satisfaction, and management modalities arranged by the cardiologists towards the relevant disease in a tertiary cardiology center.

\section{Materials and Methods}

This cross-sectional study included all consecutive patients who applied to the TM outpatient service in the cardiology department of a tertiary medical center between April $7^{\text {th }}$ and May $29^{\text {th }}, 2020$. Written informed consent was obtained from all patients being invited to the hospital after reopening of face-to-face cardiology outpatient clinic. The study was designed in accordance with the principles of the Declaration of Helsinki. It received approval from both the institutional ethics committee (no: 20-6T/40; date: 10.06.2020) and the Ministry of Health COVID-19 Scientific Research Oversight Committee.

\section{Application of Telemedicine in Outpatient Cardiology Clinic}

TM evaluation was conducted at the outpatient clinic until May 29 $9^{\text {th }}$, 2020. Telephone was decided as the most appropriate tool for telecommunication. Patients applied for TM through the hospital's electronic appointment system and entered their phone numbers to the system. Demographic features, complaints, medical history, laboratory tests, hospitalization, and treatment arrangements made by the attending cardiologist during telecommunication were retrospectively assessed by examining each patient's electronic file.

\section{Telemedicine Efficacy from a Patient's Point of View}

After reopening of face-to-face cardiology outpatient clinic, the same patients were recalled by a dedicated nurse to assess their satisfaction towards TM and to provide information about nutrition, physical activity status, and medication adherence change during the pandemic (Figure). All patients were kindly requested to reply by "Yes" or "No" for a couple of questions regarding the efficacy, satisfaction, advantages and disadvantages of TM implicated for the first time in our cardiology clinic (Supplementary Table 1). A 7-item questionnaire was formed by an investigator (E.S.) and one nurse (N.M.A.) primarily experiencing TM. Phone calls were conducted in a simple and feasible way that would not bother the patient, not extend the telephone communication time and easily give sincere answers. 


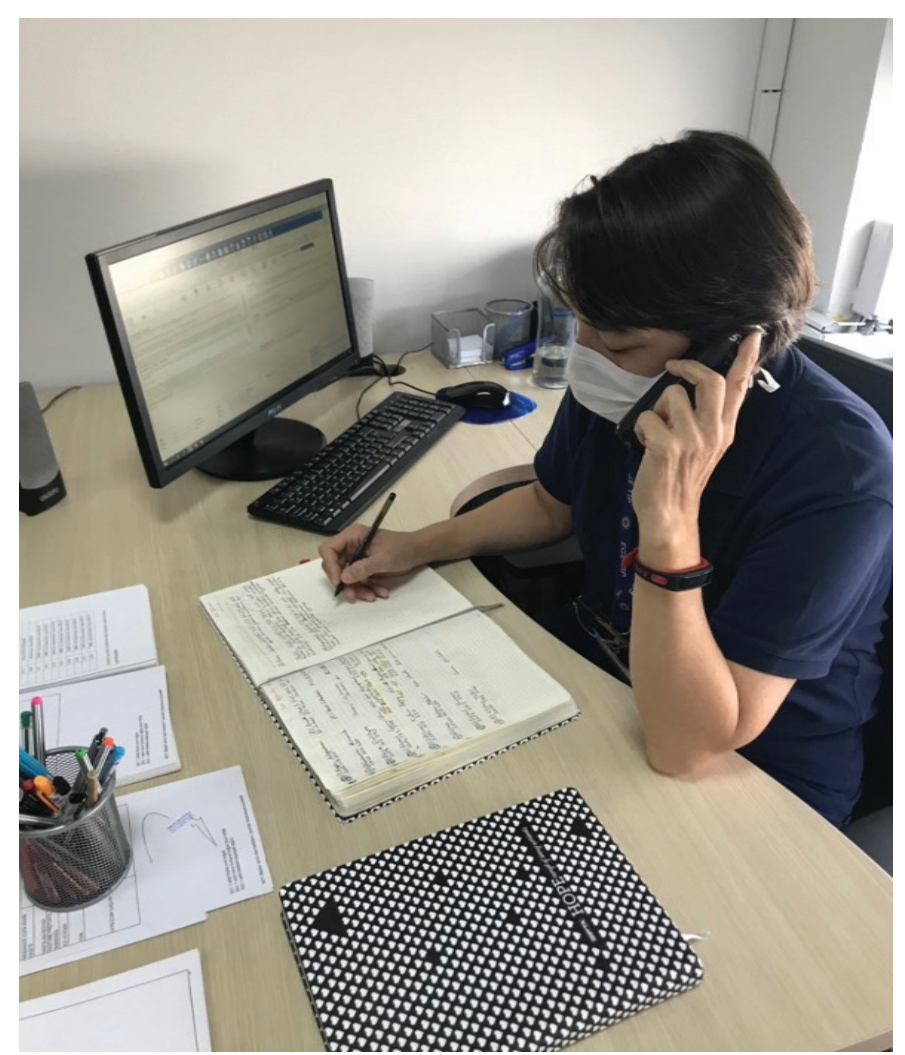

Figure. Dedicated nurse recalling the patients from the cardiology outpatient clinic for the assessment of telemedicine efficacy (01.08.2020)

\section{Statistical Analysis}

All the data were analyzed by the SPSS 25 software. The suitability of normal distribution of numerical variables was analyzed by the Shapiro-Wilk $(n<50)$ Kolmogorv-Smirnov $(\mathrm{n} \geq 50)$ test. Numerical variables were given as mean \pm standard deviation with minimal and maximal ranges. Categorical variables were presented as numbers and percentage. The relation of patient reported satisfaction was analyzed with descriptive features such as age, gender and complaints. Independent two sample t-test was used for numerical variables comparison. Categorical variables were evaluated with the chi-square test. Correlation analysis between numerical variables was done with the Spearman rank test. A p-value of $<0.05$ (two-sided) was accepted statistically significant.

\section{Results}

\section{Demographic Characteristics of Study Population}

Demographic characteristics of the study population are shown in Table 1. In nearly two-month period, a total of 140 patients had TM evaluation on the appointment day. The population was relatively old with a mean age of $69 \pm 13.75$ years and a male predominance $(53.5 \%)$. During this time, 19 patients were called more than once and reassessed by TM. Interestingly 38 patients had had a COVID-19 nasal swab test. Although all tests were negative, this feature prioritized the urgent need for a remote and efficient cardiology TM service in that period. Main complaints of application were chest pain, dyspnea and high blood pressure. About half of the population $(45.7 \%)$ had no complaints; instead, they had almost applied for a routine control, for electronic receipt and medication report prescription. The most common comorbidity was hypertension (HT) (57.8\%) and coronary artery disease (CAD) (32.1\%), followed by smoking (34.3\%) and hyperlipidemia (HLP) (26.4\%) as well. Regarding medication, beta blockers (45.7\%) and aspirin $(33.5 \%)$ were the most common used drugs before TM appointment. In addition, the rates of the use of statins, angiotensin converting enzyme inhibitor (ACEinh), and angiotensin receptor blocker (ARB) were found in a modest rate as $25 \%, 27.9 \%$, and $14.3 \%$, respectively.

\section{Physician Decision According to Patient Evaluation During TM}

Attending physicians' decision features in accordance with the evaluation of patients during TM communication are depicted in Table 2. After evaluation, 20 patients had their medical drugs rearranged. Six of them had an increase in their drug dosage due to HT and heart failure (HF). The other 14 patients had a change in their medications mostly due to uncontrolled HT. Another important cause of change of medication was exacerbation of HF with increase in body edema and dyspnea mostly prescribing furosemide, spironolactone, metoprolol, and ramipril. 
Table 1. Demographic characteristics of patients applying to cardio-telemedicine service Evaluation of patients by telemedicine application (telephone)

Age

Gender

Male $\mathrm{n}(\%)$

Female $\mathrm{n}(\%)$

Calling of patients more than once $n(\%)$

COVID-19 PCR TEST

Positive

Negative, $\mathrm{n}(\%)$

Causes of tele-medicine application, $\mathbf{n}(\%)$

Chest pain

Dyspnea

High blood pressure

Palpitations

Leg edema

No complaints

Routine control

Electronic receipt prescription

Electronic medication report prescription

Comorbidities, $\mathbf{n}(\%)$

HT

CAD

Smoking

HLP

DM

HF

Alcohol

COPD

Pacemaker

ICD

Medication of patients before telemedicine, $\mathbf{n}(\%)$

Beta-blockers

Aspirin

ACE-inh

Statin

Clopidogrel

ARB

BMI: Body mass index, COVID-19: Coronavirus disease-2019, PCR: Polymerase chain reaction, HT: Hypertension, CAD: Coronary artery disease, HLP: Hyperlipidemia, DM: Diabetes mellitus, HF: Heart failure, COPD: Chronic obstructive pulmonary disease, ICD: Intracardiac defibrillator, ACE-inh: Angiotensin converting enzyme inhibitor, ARB: Angiotensin receptor blocker, $n$ : Number

Forty-two patients were invited to outpatient clinic for face-to-face cardiac evaluation. They had multiple complaints such as angina, dyspnea, high blood pressure,
( $n=140)$

$69 \pm 13.75$

$75(53.5)$

$65(46.5)$

19 (13.5)

$38(27.1)$

0

$38(27.1)$

27 (19.3)

23 (16.4)

19 (13.5)

18 (12.8)

$8(5.7)$

64 (45.7)

$48(34.2)$

7 (5)

$9(6.4)$

81 (57.8)

45 (32.1)

48 (34.3)

37 (26.4)

29 (20.7)

27 (19.3)

22 (15.7)

7 (5)

2 (1.4)

$3(2.1)$

64 (45.7)

47 (33.5)

39 (27.9)

$35(25)$

20 (14.3)

20 (14.3)

\section{Soydan et al. Telemedicine in Cardiology Outpatient Clinic}

and advanced HF symptoms. One of them was admitted due to HF decompensation. In contrast, 18 other patients were referred to other departments due to no relevant 
Table 2. Physician decision according to cardio-telemedicine interrogation with the patients $(n=140)$

\begin{tabular}{|c|c|}
\hline Arrangement of medical drugs, $n(\%)$ & $20(14.3)$ \\
\hline Increase in medical drug dosage, $\mathrm{n}(\%)$ & $6(4.3)$ \\
\hline Metoprolol (HT) & 1 \\
\hline Ramipril (HT) & 2 \\
\hline Furosemide (HF) & 2 \\
\hline Candesartan (HT) & 1 \\
\hline Change of medical drug prescription, $n(\%)$ & $14(10)$ \\
\hline Amlodipine (HT) & 3 \\
\hline Olmesartan (HT) & 1 \\
\hline Enoxaparin (AF) & 1 \\
\hline Spironolactone (HF) & 1 \\
\hline Apixaban. (AF) & 1 \\
\hline Candesartan (HT) & 2 \\
\hline Furosemide (HF) & 1 \\
\hline Metoprolol (HF) & 1 \\
\hline Coraspin (CAD) & 1 \\
\hline Ramipril (HF) & 1 \\
\hline Perindopril (HT) & 1 \\
\hline Electronic receipt prescription, $n$ (\%) & $32(22.9)$ \\
\hline Electronic medication report, $\mathrm{n}(\%)$ & $23(16.4)$ \\
\hline Further electronic appointment, n (\%) & $21(15)$ \\
\hline Further medical tests, $\mathrm{n}(\%)$ & $43(30.7)$ \\
\hline INR measurement & 28 \\
\hline Echocardiography & 10 \\
\hline Myocardial perfusion scintigraphy & 5 \\
\hline Invitation to cardiology outpatient clinic, $\mathbf{n}(\%)$ & $42(30)$ \\
\hline Angina & 10 \\
\hline Dyspnea & 10 \\
\hline INR test & 28 \\
\hline Arrhythmia & 3 \\
\hline HF exacerbation & 7 \\
\hline Hypertension & 6 \\
\hline Hospitalization & $1(0.7)$ \\
\hline HF decompensation & 1 \\
\hline Referral to other departments & $18(12.9)$ \\
\hline
\end{tabular}

Diseases abbreviated in parentheses are the causes of medication change.

HT: Hypertension, CAD: Coronary artery disease, HF: Heart failure, AF: Atrial fibrillation, INR: International normalized ratio

cardiac complaints. Medical tests were mostly done for warfarin dose arrangement: International normalized ratio (INR). The others were echocardiography and myocardial scintigraphy for CAD differential diagnosis.

\section{Evaluation of TM}

All the patients that had applied to TM were recalled in August 2020 by telephone to make an accurate assessment of this new digital application in our department. The 
Table 3. Evaluation of patients applying to cardio-telemedicine service during follow-up ( $n=140$ )

Frequency of outpatient clinic visits before COVID-19 pandemic, $n$ (\%)

\begin{tabular}{|c|c|}
\hline First time application & $47(33.6)$ \\
\hline Once a month & $26(18.6)$ \\
\hline Once in 3 months & $15(10.7)$ \\
\hline Once in 6 months & $24(17.1)$ \\
\hline Once a year & $29(20.7)$ \\
\hline Blood pressure monitoring, $\mathrm{n}(\%)$ & $89(63.6)$ \\
\hline Systolic & $130.33 \pm 22.18(70-200)$ \\
\hline Diastolic & $78.95 \pm 13.83(40-120)$ \\
\hline Length (meter) & $1.67 \pm 0.079$ \\
\hline Weight (kilogram) & $77.86 \pm 13.21$ \\
\hline Body mass index & $27.78 \pm 4.19$ \\
\hline Impairment of nutrition status during the pandemic, $\mathrm{n}(\%)$ & $60(42.9)$ \\
\hline \multicolumn{2}{|l|}{ Alteration of nutrition habit during the pandemic, $\mathrm{n}(\%)$} \\
\hline Consume more fruits & $24(17.1)$ \\
\hline Consume more junk foods (crisps, pastry, beer nuts, biscuits etc.) & $37(26.4)$ \\
\hline Frequent meals (more than 3 times) & $25(17.9)$ \\
\hline Eating more in the late evening while watching TV & $39(27.9)$ \\
\hline Increase in salt consumption & $16(11.4)$ \\
\hline \multicolumn{2}{|l|}{ Physical exercise habit during the pandemic, $n(\%)$} \\
\hline Every day & $11(7.9)$ \\
\hline Frequently & $11(7.9)$ \\
\hline No exercise & $119(85)$ \\
\hline Decrease in physical activity during the pandemic, $n(\%)$ & $68(48.6)$ \\
\hline \multicolumn{2}{|l|}{ Medication adherence during the pandemic, $\mathrm{n}(\%)$} \\
\hline More adherent & $21(15)$ \\
\hline No change & $98(70)$ \\
\hline Not adherent & $20(14.3)$ \\
\hline
\end{tabular}

relevant features are shown in Table 3 and Table 4. They were interrogated and interestingly a high portion of them (33.6\%) had applied for the first time to our outpatient clinic, so not having the chance to get a faceto-face cardiac evaluation. During follow-up, after rearrangement of medications, only 89 patients (63.6\%) had their blood pressure continuously monitored. The mean systolic and diastolic blood pressures were found modestly normalized in a range of $130.33 \pm 22.18 \mathrm{mmHg}$ and $78.95 \pm 13.83 \mathrm{mmHg}$, respectively. On the other hand, about half of patients (42.9\%) had an impairment regarding physical and nutritional status during the pandemic period, showing modestly increased body mass index in an overweight range (27.78 \pm 4.19$)$. They began to eat more than 3 meals a day with the consumption of more junk foods such as crisps, pastry, beer nuts etc. especially in the late evening hours. A high portion $(85 \%)$ as well did not make any exercise at all during that period and about half of the interrogated patients (48.6\%) expressed a decrease in physical activity. There was no evident change in medication adherence in only 20 patients having some challenges remembering the time 
Table 4. Evaluation of cardio-telemedicine by patients $(n=140)$

Prevention of unnecessary hospital application, $\mathbf{n}(\%)$

Hospital appointment easier than before, $n(\%)$

Waiting time shortened in case of hospital referral, $\mathbf{n}(\%)$

Easy hospital appointment through telemedicine application, $\mathbf{n}(\%)$

Time for cardio-telemedicine appointment (days)

Time of cardio-telemedicine call (minutes)

Difficulty in expressing the complains during cardio-telemedicine call, $\mathbf{n}(\%)$

Willingness for cardio-telemedicine continuation after the pandemic, $\mathbf{n}(\%)$

Preference of telemedicine instead of face-to-face outpatient in terms of easy complaint expression and understanding of medication rearrangement, $\mathbf{n}(\%)$

Satisfaction of patients from the cardio-telemedicine service, $n(\%)$

Non-satisfaction of patients from the cardio-telemedicine service, $n(\%)$

Cause of non-satisfaction

Not referral to hospital

No need for further tests

Relation of patient reported satisfaction with demographic features

Statistics

Age

Gender

Angina

Dyspnea

High blood pressure

Palpitations

Asymptomatic

n: Number
$135(96.4)$

$133(95)$

$129(92.1)$

$130(92.8)$

$2.53 \pm 1.39(1-8)$

$6.68 \pm 5.0(3-20)$

2 (1.4)

$123(87.8)$

75 (53.6)

$131(93.6)$

$9(6.4)$

5

4

Rho: $0.042, p=0.221$

$p=0.692$

$p=0.699$

$p=0.078$

$p=0.053$

$p=0.521$

$p=0.915$

Supplementary Table 1. 7-item questionnaire assessing the efficacy of telemedicine (all patients were requested to answer as yes or no)

1. Were you satisfied with the TM for continuing of healthcare in the COVID-19 pandemic?

2. If no, please state the cause

3. Did the TM application prevent unnecessary hospital visit?

4. In case of hospital referral, was the time of waiting shortened?

5. Was it easy to get an appointment for TM evaluation

6. Did you have any difficulty expressing your complaints during TM call?

7. Do you prefer TM be continued after the pandemic?

8. Which service would you prefer the most in terms of easy expression of your complaints: Face-to-face or TM?

TM: Telemedicine, COVID-19: Coronavirus disease-2019 
and dose of their medications. However, almost all (93.6 $\%$ ) of the patients were very satisfied and preferred TM instead of face-to-face outpatient clinic service in terms of easy complaint expression and easy understanding of medication rearrangement $(53.6 \%)$. Satisfaction was found to be an important feedback for this new emerging digital health care irrespective of demographic features such as age, gender and complaints. Instead, the causes of nonsatisfaction were mainly expressed by younger patients willing further tests and referral to hospital.

Most of the patients agreed with the idea that the new digital health tool, TM, was efficient in the prevention of unnecessary hospital application, provided easier hospital appointment than before the pandemic, shortened time of waiting in case of hospital referral and it should continue after the pandemic as well. In addition, they all took attention to the time (6.68 \pm 5.0 minutes) spent during TM communication, which was used in a sufficient and efficient way.

\section{Discussion}

\section{Implication of Telemedicine in Cardiology Outpatient Clinic}

In order to prevent such steep increase in infection transmission of COVID-19 in both the patients and healthcare providers, remote control via digital health tools should be urgently implemented in the routine of outpatient clinic services ${ }^{(8)}$. Although TM has been considered by the World Health Organization as the alternative way for remote patients uncapable of coming to the medical facilities, it has become a potential alternative in the COVID-19 era in terms of efficacy of healthcare delivery and patient satisfaction ${ }^{(9,10)}$. In the light of these lifesaving alternative ways for continuing of healthcare delivery, we experienced for the first time TM practice during the extraordinary time of COVID-19 pandemic in our cardiology outpatient clinic.

The older age of the population with multiple cardiac risk factors applying to TM showed that the new digital health system was introduced in a very appropriate time where all the patients had need for the continuation of healthcare service. Although, it was a first-time experience for us, telecommunication by telephone provided high confidence in both the patient and the physician by clearly expressing themselves. Possible causes of providing high confidence during telecommunication were empathy expressing behaviors. For instance, patients expressed that listening to them and letting them guide the conversation was one of the evident clues noticed in most of the communications. Afterwards, we had 19 patients that were called for TM evaluation more than once, a feature that provides confidence and the patient not to be felt forgotten. On the other hand, telephone contact with cardiac patients before a face-to-face examination could motivate their self-consciousness of their disease. In this way, patients can become more conscious in the self-management of their cardiac disease by monitoring of their diet, blood pressure, heart rate, body weight and glucose at home.

\section{Management of Cardiac Diseases by Telemedicine}

Studies have shown that HT can be easily and effectively managed via TM. This could eliminate the 'white coat hypertension' phenomena, thus appropriately provide the necessary dose of antihypertensive drug ${ }^{(11)}$. Indeed, our experience with TM provided efficacy in HT management with good blood pressure monitoring during follow up. Although we did not have a high number of patients with HF, a guideline-based therapy was efficiently provided and a good feedback was obtained from the patients. This simple telecommunication model for a short time was found to be in accordance with the European Society of Cardiology consensus on the role of TM in HF patients showing a good medication management and high alertness for HF exacerbation ${ }^{(6)}$.

\section{Evaluation of Nutritional and Physical Status of Study Population During the Pandemic}

The high and fast rate of infection spread made people fear from going outside so almost all the patients evaluated in this study were prisoned at home for a long time. During 
this period, we queried them about any change of nutritional and physical status. Although patients were acknowledged about proper diet and healthy lifestyle modification during TM service, interestingly, about $43 \%$ of them stated an impairment in their nutritional status during the pandemic. Especially, spending a long time staying at home was the leading cause to trigger consumption of more junk food such as crisps, pastry or beer nuts. The only entertainment tool was the television making them unwittingly increase the number of meals and more frequently eat in the late hours of the evening.

It has been demonstrated that older adults ( $>60$ years of age) are at the greatest risk of mortality due to the COVID-19 disease and consequently they represent the most affected population from the measures of social isolation $^{(12,13)}$. Similar to our study population, preventive measures can expose older people to an impaired nutritional status by pushing them towards overeating mostly fast foods rich in fat, sugar and salt ${ }^{(14)}$. Undernutrition as well is another challenging issue due to socioeconomic problems commonly encountered among older people, which can lead to a worse prognosis during COVID-19 disease. One of the pioneer services was the "Great Plates Delivered" program to supply three healthy meals to older people at risk for infection, which promoted awareness of fighting infection with balanced and healthy regular meal habit ${ }^{(15)}$. Moreover, a balanced and nutritious diet has been shown to be one of the keys for a better prognosis in older adults with COVID-19 disease, so the introduction of educational programs and social facilities toward promoting healthy nutrition should be encouraged ${ }^{(16,17)}$.

\section{Medical Avoidance During the Pandemic}

Medical care avoidance has been demonstrated as an unacceptable cause of decreased and late acute coronary syndrome admissions during the pandemic, thus leading to increased cardiac events ${ }^{(18)}$. This is of paramount importance as most people are frightened from contracting the COVID-19 infection and so avoid seeking medical care. However, our study showed that easy implementation of TM can provide a new way for efficacious health care continuation by applying triage for patients necessitating medical assistance and as a result overcome medical avoidance.

\section{Telemedicine Efficacy from a Patent's Point of View}

Satisfaction of patients, especially older ones, with TM has been demonstrated with video visits during the COVID-19 pandemic, a very important point underscoring the truly need of seniors for healthcare delivery ${ }^{(19)}$. Similar to this largest study, our relatively old population showed high satisfaction rates irrespective of demographic factors and complaints. This promising feature can be fundamental for future implication of TM in a routine basis for cardiac patients' follow-up.

\section{Study Limitations}

Despite having a small sample size, this study demonstrated the first experience of TM implication in the beginning of the pandemic in a tertiary medical center. It could have been more sophisticated and efficacious if other tools like video communication and email feedback would have been incorporated into the digital health service. The attending physicians were not interrogated in terms of satisfaction in order to find out the practical difficulties experienced by them. As the infrastructure of medical facilities were not ready at the beginning of the pandemic, telephone was the only simple and most reachable tool in performing TM. The TM was conducted contemporarily with the onset of the lock down of the population, so we did not make any comparison with the post lock down period. In addition, lock down measures affected mostly the older people reflecting the cause of the older age of our study population. However, we did demonstrate that the older patients could effectively use the telephone for reaching medical assistance via TM. The lack of use of a validated TM efficacy questionnaire is another limitation of the study. However, as the study was conducted in the very early period of the pandemic, there were no validated questionnaires constructed for assessing the efficacy of the TM or patient satisfaction. 
However, it should be highlighted that this is the first study in Turkey describing digital health TM implication during the pandemic. Therefore, it should serve as a fundamental for constructing a more sophisticated digital health system in the future of healthcare system.

\section{Conclusion}

TM is a potential alternative for continuing healthcare delivery to most of the cardiac patients necessitating rigorous follow-up during the COVID-19 pandemic. Administrative protocols with training of healthcare providers and ethical and legislative criteria are needed for future digital health introduction in routine clinical practice. All these implications should be rearranged according to specific groups of cardiac patients, especially those with arrhythmic and advanced heart failure, integrating accurate criteria for future benefit of digitalization in modern medicine.

\section{Acknowledgements}

We want to thank nurse Nuray Memişoğlu Akgül for helping in collecting data and telecommunicating with the study population.

\section{Ethics}

Ethics Committee Approval: The study was designed in accordance with the principles of the declaration of Helsinki. It received approval from both the Institutional Ethics Committee (20-6T/40; 10.06.2020) and the Ministry of Health COVID-19 Scientific Research Oversight Committee.

Informed Consent: Informed consent was taken from all the patients.

Peer-review: Externally peer-reviewed.

\section{Authorship Contributions}

Surgical and Medical Practices: E.S., Concept: E.S., M.Z., Design: E.S., M.Z., Data Collection or Processing: E.S., Analysis or Interpretation: E.S., M.K., M.Z., Literature Search: E.S., M.K., M.Z., Writing: E.S., M.K., M.Z.

Soydan et al. Telemedicine in Cardiology Outpatient Clinic
Conflict of Interest: The authors declare that they have no conflict of interest.

Financial Disclosure: This research received no grant from any funding agency in the public, commercial or notfor-profit sectors.

\section{References}

1. Kaushik A, Patel S, Dubey K. Digital cardiovascular care in COVID-19 pandemic: A potential alternative? J Card Surg 2020;35:3545-50.

2. Aktoz M, Altay H, Aslanger E, et al. Türk Kardiyoloji Derneği Uzlaşı Raporu: COVID-19 Pandemisi ve Kardiyovasküler Hastalıklar Konusunda Bilinmesi Gerekenler (25 Mart 2020) [Consensus Report from Turkish Society of Cardiology: COVID-19 and Cardiovascular Diseases. What cardiologists should know. Turk Kardiyol Dern Ars 2020;48(Suppl 1):1-48.

3. Du RH, Liang LR, Yang CQ, et al. Predictors of mortality for patients with COVID-19 pneumonia caused by SARS-CoV-2: a prospective cohort study. Eur Respir J 2020;55:2000524.

4. Madjid M, Safavi-Naeini P, Solomon SD, Vardeny O. Potential Effects of Coronaviruses on the Cardiovascular System: A Review. JAMA Cardiol 2020;5:831-40.

5. WHO Guideline: recommendations on digital interventions for health system strengthening. Geneva: World Health Organization; 2019. Available at: https://www.who.int/reproductivehealth/publications/digitalinterventions-health-system-strengthening/en/

6. Seferovic PM, Ponikowski P, Anker SD, et al. Clinical practice update on heart failure 2019: pharmacotherapy, procedures, devices and patient management. An expert consensus meeting report of the Heart Failure Association of the European Society of Cardiology. Eur J Heart Fail 2019;21:1169-86.

7. Koehler F, Koehler K, Deckwart O, et al. Telemedical Interventional Management in Heart Failure II (TIM-HF2), a randomised, controlled trial investigating the impact of telemedicine on unplanned cardiovascular hospitalisations and mortality in heart failure patients: study design and description of the intervention. Eur J Heart Fail 2018;20:1485-93.

8. Kumar P, Huda F, Basu S. Telemedicine in the COVID-19 era: the new normal. Eur Surg 2020:1-2.

9. WHO Global Observatory for eHealth. (2010). Telemedicine: opportunities and developments in Member States: report on the second global survey on eHealth [Internet]. World Health Organization. Available at: https://apps. who.int/iris/handle/10665/44497

10. Polinski JM, Barker T, Gagliano N, Sussman A, Brennan TA, Shrank WH. Patients' Satisfaction with and Preference for Telehealth Visits. J Gen Intern Med 2016;31:269-75.

11. Milani RV, Lavie CJ, Bober RM, Milani AR, Ventura HO. Improving Hypertension Control and Patient Engagement Using Digital Tools. Am J Med 2017;130:14-20.

12. Barazzoni R, Bischoff SC, Breda J, et al. ESPEN expert statements and practical guidance for nutritional management of individuals with SARSCoV-2 infection. Clin Nutr 2020;39:1631-8. 
13. Onder G, Rezza G, Brusaferro S. Case-Fatality Rate and Characteristics of Patients Dying in Relation to COVID-19 in Italy. JAMA 2020;323:1775-6.

14. Yılmaz C, Gökmen V. Neuroactive compounds in foods: Occurrence, mechanism and potential health effects. Food Res Int 2020;128:108744.

15. California State Government (United States). Great plates delivered: home meals for seniors: coronavirus COVID-19 response. Sacramento: California State Government; 2020 [cited 2020 Dec 11]. Available from: https://files. covid19.ca.gov/pdf/wp/great-plates-delivered-program-guidance.pdf

16. Ceolin G, Moreira JD, Mendes BC, Schroeder J, Di Pietro PF, Rieger DK. Nutritional challenges in older adults during the COVD-19 pandemic. Rev Nutr 2020;33: e200174.
17. Jayawardena R, Sooriyaarachchi $\mathrm{P}$, Chourdakis $\mathrm{M}$, Jeewandara $\mathrm{C}$, Ranasinghe P. Enhancing immunity in viral infections, with special emphasis on COVID-19: A review. Diabetes Metab Syndr 2020;14:367-82.

18. Erol MK, Kayıkçığlu M, Kılıçkap M, et al. Treatment delays and inhospital outcomes in acute myocardial infarction during the COVID-19 pandemic: A nationwide study. Anatol J Cardiol 2020;24:334-42.

19. Ramaswamy A, Yu M, Drangsholt S, et al. Patient Satisfaction With Telemedicine During the COVID-19 Pandemic: Retrospective Cohort Study. J Med Internet Res. 2020;22:20786. 